\title{
Two single nucleotide polymorphisms in the GDF5 gene are associated with development dysplasia of the hip in Chinese female population
}

\author{
ZHAO LiXi $^{1,2}$, PAN Hong ${ }^{1,2}$, WANG Jing ${ }^{1,2}$, CHENG Zhi ${ }^{1,2}$, CHENG LongFei ${ }^{1,2}$, \\ WANG BinBin ${ }^{1,2^{*}} \&$ MA Xu ${ }^{1,2,3^{*}}$ \\ ${ }^{1}$ Graduate School of Peking Union Medical College, Beijing 100730, China; \\ ${ }^{2}$ National Research Institute for Family Planning, Beijing 100081, China; \\ ${ }^{3}$ World Health Organization Collaborating Centre for Research in Human Reproduction, Beijing 100081, China
}

Received March 22, 2013; accepted May 21, 2013; published online October 9, 2013

Citation: Zhao L X, Pan H, Wang J, et al. Two single nucleotide polymorphisms in the GDF5 gene are associated with development dysplasia of the hip in Chinese female population. Sci China Life Sci, 2013, 56: 1063-1065, doi: 10.1007/s11427-013-4514-0

Development dysplasia of the hip (DDH) is one of the most common hip defects in newborns. The main phenotypes of the disease are partial or whole femoral head extrusion from the acetabulum [1]. Because the frequency of DDH in monozygotic twins was higher than that in dizygotic twins, and the affected individuals were often in the same family [2], we are focusing on the genetic factor in the present study.

Growth differentiation factor 5 (GDF5) belongs to the transforming growth factor (TGF)-beta super-family of proteins. It plays an important role in embryonic limb development, especially in the formation of cartilage and articular cavities. In early skeletal development, it promotes the aggregation and adherence of protochondrial cells; and in the late period, it stimulates the proliferation and hypertrophy of cartilage cells [3].

In the present study, we performed an association study between GDF5 and DDH development in a female Han Chinese population by genotyping GDF5 single nucleotide polymorphisms (SNPs) with the aim of determining the relationship between $G D F 5$ and DDH.

We selected 192 unrelated female Han Chinese DDH patients (mean age $\pm \mathrm{SD}=5.21 \pm 3.16$ months) and 191 unrelated, healthy, ethnically-matched female controls (mean age $\pm \mathrm{SD}=$

*Corresponding author (email: NICGR@263.net; binbincas@ hotmail.com)
6.22 \pm 3.85 months) from Tianjin Hospital, Tianjin, China; there were only 11 bilateral DDH patients, while the other patients were unilateral. All patients had been diagnosed with DDH using ultrasound and radiography according to the clinical criteria: acetabulum angle increased $\left(>25^{\circ}\right)$, center-edge $(\mathrm{CE})$ angle decreased $\left(<20^{\circ}\right)$, sharp angle increased $\left(>45^{\circ}\right)$, the Shenton's line disrupted, and the acetabular head index (AHl) was not in the scope of $84 \%-85 \%$. Those patients with systemic syndrome were excluded from the study. All selected DDH cases were sporadic and there was no family history of the disease. Informed consent for study participation was obtained from the parent/guardian of each patient. The study protocol is in accordance with the ethical guidelines of the 1975 Declaration of Helsinki and is approved by the Ethics Committee of the National Research Institute for Family Planning.

Genomic DNA from peripheral blood leukocytes of DDH patients and controls was extracted by a QIAamp blood kit (Qiagen, Hilden, Germany) according to the manufacturer's instructions.

The tag SNPs were selected according to Phase II of the International HapMap project. We selected tag SNPs considering their pairwise tagging algorithms; $r^{2}$ exceeded 0.8 for all known SNPs with a minor allele frequency (MAF)> $5 \%$ [4]. 
The PCR primers for the association study were designed using Sequenom MassARRAY® designer software. Phenotypic analysis was performed by matrix-assisted laser desorption/ionization time-of-flight mass spectrometry (MALDITOF MS) after PCR product purification.

Allele frequencies and genotype distributions of patients and controls were analyzed by Pearson $\chi$-square tests using the SPSS10.0 software. According to the Bonferroni correction, the estimated statistical significance was $P<0.025$. At last, the statistical power was calculated by power and precision v4 software.

Two tag SNPs, rs224332 and rs224333, were identified using the methods described above, and were analyzed for genotyping. Both SNPs were located in the same intron of GDF5. The distributions of the two SNPs in both patient and control groups were in Hardy-Weinberg equilibrium $(P \geqslant 0.05)$. The statistical power value was 0.85 .

For SNP rs224332, significant differences in both allele frequency and genotype distribution were observed between patients and controls $\left(\chi^{2}=10.65, \mathrm{~d} f=1, P=0.001\right.$ and $\chi^{2}=$ $11.03, \mathrm{~d} f=2, P=0.004$, respectively). The $\mathrm{C}$ allele was indicated to be a risk factor for DDH development because of its higher frequency in patients $(94.79 \%)$. Genotype distribution analysis indicated a recessive mode of transmission $\left(\chi^{2}=9.33, \mathrm{~d} f=1, P=0.001\right)$ (Table 1). Significant differences existed between the $\mathrm{CC}$ genotype and the other two genotypes combined in the rs224332 SNP.

SNP rs224333 also showed an association with DDH development (Table 1). Significant differences were observed in allele frequencies and genotype distributions in patient and control groups $\left(\chi^{2}=7.68, \mathrm{~d} f=1, P=0.006\right.$ and $\chi^{2}=12.10, \mathrm{~d} f=2, P=0.002$, respectively). The $\mathrm{G}$ allele was shown to be a risk factor for DDH development, and genotype distribution analysis suggested a dominant mode of inheritance $\left(\chi^{2}=10.88, \mathrm{~d} f=1, P=0.001\right)$. The GG genotype frequency of the rs224333 SNP was significantly higher than that of the other two genotypes combined.
Previously, other SNPs have been shown to be associated with DDH development $[5,6]$, but this is the first time to suggest association between another two SNPs (rs224332 and rs224333) and DDH.

In the database of the 1000 Genomes Project (http://www. 1000 genomes.org), $\mathrm{C}$ is the common allele of rs224332 associated with DDH development, with a frequency of $88 \%$ in all populations and $85 \%$ in the ASN database. Similarly, $\mathrm{G}$ is the common allele of rs224333 associated with DDH development. The frequencies of the $\mathrm{G}$ allele are $54 \%$ and $76 \%$ in all populations and ASN, respectively. Thus, our association study provides another example of a common allele that could be related to disease development [7].

GDF5 levels vary during joint formation. The protein is first detected in the developing limb joint on day 11.5 days post-conception (dpc) and continues to be expressed on days 12.5-14.5, decreasing after day $15.5 \mathrm{dpc}$ [8]. GDF5 affects skeletal development by stimulating the development, growth and maturation of cartilage, and restricts synovial joint formation by regulating the expression of joint-specific markers [9]. Thus, GDF5 is an important factor in regulating joint formation and hip development.

Besides, two pathological lesions (limbus and neolimbu) which were characterized by fibrous and fibrocartilaginous hyperplasia were detected in DDH patients [10]. Motta [11] showed that neolimbus existed in $25 \mathrm{DDH}$ patients but was absent from 300 controls. Considering the effects of GDF5 on cartilage development, it is conceivable that abnormal GDF5 is related to these pathological lesions, and thus contributes to the process of DDH development.

In conclusion, we have established an association between two SNPs (rs224332 and rs224333) of GDF5 and DDH development in a female Chinese population. Our findings indicate that $G D F 5$ may be a candidate gene for DDH development, and are helpful in understanding the genetic pathogenesis of DDH.

Table 1 Allele frequencies and genotype distributions of GDF5 tag SNPs

\begin{tabular}{|c|c|c|c|c|c|c|}
\hline \multirow[t]{2}{*}{ Single marker } & & \multicolumn{2}{|c|}{ Allele } & \multicolumn{3}{|c|}{ Genotype } \\
\hline & & A (freq) & $\mathrm{C}($ freq) & $\mathrm{A} / \mathrm{A}$ (freq) & $\mathrm{A} / \mathrm{C}$ (freq) & $\mathrm{C} / \mathrm{C}$ (freq) \\
\hline & Case & $20(5.21 \%)$ & $364(94.79 \%)$ & $0(0 \%)$ & $20(10.42 \%)$ & $172(89.58 \%)$ \\
\hline \multirow[t]{5}{*}{ rs 224332} & Control & $45(11.78 \%)$ & $337(88.22 \%)$ & $2(1.05 \%)$ & $41(21.47 \%)$ & $148(77.49 \%)$ \\
\hline & & \multicolumn{2}{|c|}{$\chi^{2}=10.65, \mathrm{~d} f=1, P=0.001$} & \multicolumn{3}{|c|}{$\chi^{2}=11.03, \mathrm{~d} f=2, P=0.004$} \\
\hline & \multicolumn{6}{|c|}{$\chi^{2}=9.33, \mathrm{~d} f=1, P=0.001$ by recessive mode } \\
\hline & & A (freq) & $\mathrm{G}$ (freq) & $\mathrm{A} / \mathrm{A}$ (freq) & $\mathrm{A} / \mathrm{G}$ (freq) & $\mathrm{G} / \mathrm{G}$ (freq) \\
\hline & Case & $59(15.45 \%)$ & $323(84.55 \%)$ & $1(0.52 \%)$ & $57(29.84 \%)$ & $133(69.63 \%)$ \\
\hline \multirow[t]{3}{*}{ rs 224333} & Control & $88(23.40 \%)$ & $288(76.60 \%)$ & $13(6.91 \%)$ & $62(32.98 \%)$ & $113(60.11 \%)$ \\
\hline & & \multicolumn{2}{|c|}{$\chi^{2}=7.68, \mathrm{~d} f=1, P=0.006$} & \multicolumn{3}{|c|}{$\chi^{2}=12.10, \mathrm{~d} f=2, P=0.002$} \\
\hline & & & $\chi^{2}=10.88$, & 001 by don & & \\
\hline
\end{tabular}


This work was supported by the National Basic Research Program of China (2010CB529500), the International Cooperative Research Program of Translational Medicine for Congenital Heart Defects, and Ministry of Science and Technology of China (2011DFA33120). The authors declare that they have no competing interests.

1 Hurley A. DDH: causes and examination. Community Pract, 2009, 82: 36-37

2 Wilkinson J A. Etiologic factors in congenital displacement of the hip and myelodysplasia. Clin Orthop Relat Res, 1992, 281: 75-83

3 Coleman C M, Tuan R S. Functional role of growth/differentiation factor 5 in chondrogenesis of limb mesenchymal cells. Mech Dev, 2003, 120: 823-836

4 de Bakker P I, Yelensky R, Pe'er I, et al. Efficiency and power in genetic association studies. Nat Genet, 2005, 37: 1217-1223

5 Dai J, Shi D, Zhu P, et al. Association of a single nucleotide polymorphism in growth differentiate factor 5 with congenital dysplasia of the hip: a case-control study. Arthritis Res Ther, 2008, 10: R126

6 Rouault K, Scotet V, Autret S, et al. Evidence of association between GDF5 polymorphisms and congenital dislocation of the hip in a Caucasian population, Osteoarthritis Cartilage, 2010, 18: 1144-1149

7 Bodmer W, Bonilla C. Common and rare variants in multifactorial susceptibility to common diseases. Nat Genet, 2008, 40: 695-701

8 Storm E E, Kingsley D M. Joint patterning defects caused by single and double mutations in members of the bone morphogenetic protein (BMP) family. Development, 1996, 122: 3969-3979

9 Storm E E, Kingsley D M. GDF5 coordinates bone and joint formation during digit development. Dev Biol, 1999, 209: 11-27

10 Landa J, Benke M, Feldman D S. The limbus and the neolimbus in developmental dysplasia of the hip. Clin Orthop Relat Res, 2008, 466: 776-781

11 Motta F. Ultrasonography in the diagnosis of congenital hip dysplasia in the newborn. Int Orthop, 1989, 13: 29-31

Open Access This article is distributed under the terms of the Creative Commons Attribution License which permits any use, distribution, and reproduction in any medium, provided the original author(s) and source are credited. 